\title{
Large-Scale Tests of the DGP Model
}

\author{
Yong-Seon Song, ${ }^{1,2}$ Ignacy Sawicki, ${ }^{1,3}$ and Wayne $\mathrm{Hu}^{1,2}{ }^{2}$. \\ ${ }^{1}$ Kavli Institute for Cosmological Physics, Enrico Fermi Institute, University of Chicago, Chicago IL 60637 \\ 2 Department of Astronomy 8 Astrophysics, University of Chicago, Chicago IL 60637 \\ 3 Department of Physics, University of Chicago, Chicago IL 60637
}

(Dated: October 4, 2018)

\begin{abstract}
The self-accelerating braneworld model (DGP) can be tested from measurements of the expansion history of the universe and the formation of structure. Current constraints on the expansion history from supernova luminosity distances, the CMB, and the Hubble constant exclude the simplest flat DGP model at about $3 \sigma$. Even including spatial curvature, best-fit open DGP model is a marginally poorer fit to the data than flat $\Lambda$ CDM. Moreover, its substantially different expansion history raises serious challenges for the model from structure formation. A dark-energy model with the same expansion history would predict a highly significant discrepancy with the baryon oscillation measurement due the high Hubble constant required. For the DGP model to satisfy this constraint new non-linear phenomena must correct this discrepancy. Likewise the large enhancement of CMB anisotropies at the lowest multipoles due to the ISW effect would require either a cut off in the initial power or new phenomena at the cross over scale. A prediction that is robust to both possibilities is that high-redshift galaxies should be substantially correlated with the CMB through the ISW effect. This correlation should provide a sharp test of the DGP model in the future.
\end{abstract}

PACS numbers:

\section{INTRODUCTION}

Cosmic acceleration may arise either from a new form of energy density, dubbed dark energy, or from a modification of gravity on cosmological scales. Dvali, Gabadadze and Porrati (DGP) [1] have proposed a braneworld modification of gravity to explain acceleration (see [2] for a recent review). In this model our universe is a $(3+1)$-dimensional brane embedded in an infinite Minkowski bulk. The cosmological solution of this theory exhibits self-acceleration on the brane classically [3, 4].

Various cosmological probes which can distinguish DGP from dark energy models (DE) have been investigated in the literature $5,6,6,8,8,9,10,11,12,13,14$, 15, 16]. These come in two classes: those that test DGP modifications of the expansion history and those that test changes in how cosmological structures are formed. The advantage of the former class is that the predictions of the DGP model are well understood theoretically. The main disadvantage is that they currently lack the power to distinguish the DGP model from its dark energy counterpart the $\Lambda \mathrm{CDM}$ model at high significance. Moreover, expansion rate tests cannot distinguish between the DGP model and an arbitrary form of smooth dark energy that is constructed to mimic it in the background. Structure formation tests on the other hand can in principle solve both these problems. However due to complexities in the theory they currently can only be rigorously implemented on a rather small range of scales.

In this paper, we examine the combination of these two types of tests. We employ three tests of the expan-

*Electronic address: ysong@cfcp.uchicago.edu sion history: the angular diameter distance to recombination from the three-year WMAP data [17], luminosity distance to high-redshift supernovae (SNGold[18] and SNLS [19]) and the local expansion rate from Hubble constant measurements. These tests do in fact strongly disfavor the simplest version of the DGP model: a modification of gravity in a spatially flat cosmology with no bulk cosmological constant. The best fit DGP model has a significant negative curvature and a high Hubble constant. Even with the addition of spatial curvature, it is a slightly poorer fit to the data than flat $\Lambda$ CDM.

The best fit DGP model yields an expansion history that is strikingly different from flat $\Lambda$ CDM. The dark energy model that mimics this expansion history has negative curvature and a dark energy equation of state that increases sharply with redshift. In the dark energy context such an expansion history would be strongly excluded by structure formation tests in weakly to strongly non-linear regime. These include the baryon acoustic oscillations (BAO) in galaxy clustering [16, 20], the abundance of galaxy clusters, and fluctuations in the Lyman$\alpha$ forest. While these probes likely also test the DGP model, none of these phenomena are sufficiently well understood in the model to provide a definitive test. Indeed the presence of ghost degrees of freedom in the DGP model around a de-Sitter background [21, 22, 23, 24] require that the nature of gravitational interactions change dramatically in the strongly-coupled non-linear regime for the model to remain a viable explanation of cosmic acceleration [25].

Under this assumption, structure formation in DGP is currently well understood on scales between the Hubble scale and the scale radius of a typical dark matter halo. Large scale CMB anisotropy is substantially enhanced by the Integrated Sachs-Wolfe (ISW) effect. At the lowest multipoles, this excess poses a significant challenge for 
the model. Possible resolutions of this problem still leave a robust prediction and sharp test of the DGP model: high redshift galaxies should be substantially correlated with the CMB.

\section{EXPANSION HISTORY}

\section{A. Supernovae, CMB and $H_{0}$}

The expansion history of a DGP model is determined by the usual Friedman-Robertson-Walker parameters and the crossover distance. The crossover distance is defined as the ratio of 5-dimensional to 4-dimensional Planck mass scales

$$
r_{\mathrm{c}}=\frac{M_{\mathrm{Pl}}^{(4) 2}}{2 M_{\mathrm{Pl}}^{(5) 3}} .
$$

It is a free parameter of the theory. If $r_{\mathrm{c}}$ is close to the current horizon scale, the acceleration of cosmic expansion is replicated without dark energy. Gravity on scales much less than $r_{\mathrm{c}}$ does not penetrate into the bulk significantly, and exhibits the usual Newtonian potential falling as the inverse distance. In the DGP model, as the Hubble scale approaches $r_{c}$, the universe enters an accelerating, asymptotically de-Sitter phase despite the absence of a cosmological constant.

The main evidence for cosmic acceleration is geometrical and based on the luminosity distance to high-redshift supernovae (SN) [18, 26, 27, 28] and the angular diameter distance to the recombination epoch of the CMB (e.g. [29]). Both distances are given as usual by the (comoving) angular diameter distance in units of $H_{0}^{-1}$

$$
D(z)=\frac{1}{\sqrt{\Omega_{k}}} \sinh \left(r(z) \sqrt{\Omega_{k}}\right)
$$

where $r(z)$ is

$$
r(z)=\int_{0}^{z} \frac{d z^{\prime}}{E\left(z^{\prime}\right)}
$$

$E(z)$ is the Hubble parameter in units of the Hubble constant. The DGP model modifies the Friedman equation or the relationship between $E(z)$ and the energy densities of matter and radiation in units of the critical density

$$
\begin{aligned}
E^{2} & \equiv \frac{H^{2}}{H_{0}^{2}} \\
& =\Omega_{k} a^{-2}+\left(\sqrt{\Omega_{\mathrm{rc}}}+\sqrt{\Omega_{\mathrm{rc}}+\Omega_{m} a^{-3}+\Omega_{r} a^{-4}}\right)^{2},
\end{aligned}
$$

where $H_{0}=2997.9 \mathrm{~h} \mathrm{Mpc}^{-1}$. Here the scale factor $a=$ $1 /(1+z), \Omega_{\mathrm{rc}}\left(=1 / 4 r_{\mathrm{c}}^{2} H_{0}^{2}\right)$ is given by

$$
\Omega_{\mathrm{rc}}=\frac{\left(1-\Omega_{k}-\Omega_{m}-\Omega_{r}\right)^{2}}{4\left(1-\Omega_{k}\right)} .
$$

We implement the SN constraints from the "gold" SN data set of [18] and the SNLS set of [19]. For the CMB, we fix the distance to recombination at $z_{\mathrm{lss}}=1088_{-2}^{+1}$ through the measurement of the acoustic peak scale $l_{A}=302_{-1.4}^{+0.9}$ and its length calibration through the matter density $\Omega_{m} h^{2}=0.1268_{-0.0095}^{+0.0072}[29]$. Note that the SN are a relative distance indicator and hence constrain $D$ whereas the CMB is an absolute distance measure which constrains $D / h$.

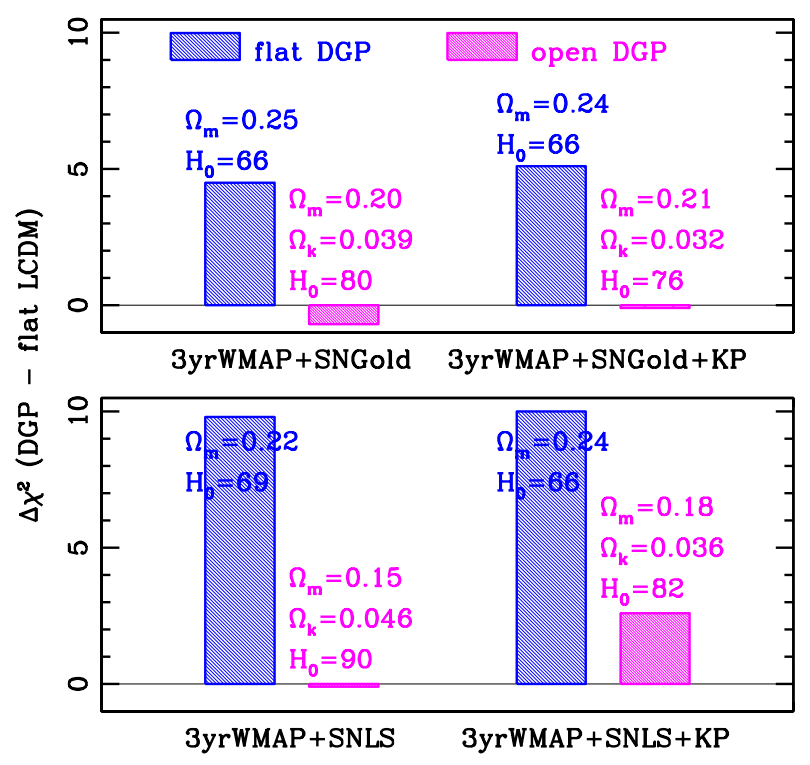

FIG. 1: The $\Delta \chi^{2}$ between the best fit flat and open DGP versus that of flat $\Lambda$ CDM model. SNGold supernova (SN) data set is used in the top panel and SNLS SN data set is used in the bottom panel. The DGP model requires curvature and a high Hubble constant. With the addition of Key Project (KP) direct Hubble constant measurements open DGP is a marginally poorer fit to the data than flat $\Lambda \mathrm{CDM}$.

Like flat $\Lambda \mathrm{CDM}$ (f $\Lambda \mathrm{CDM})$, flat DGP has a single parameter $\Omega_{m}$ that is over-constrained by the distances to recombination, $\Omega_{m} h^{2}$, and high-redshift supernovae. Unlike $\mathrm{f} \Lambda \mathrm{CDM}$, flat DGP is not a good fit to these data. In DGP cosmologies, the SN favor a lower $\Omega_{m}$ than in $\mathrm{f} \Lambda \mathrm{CDM}$ resulting in a shorter angular diameter distance to the CMB than is measured by WMAP. For the SNGold data set the excess $\chi^{2}$ over $\mathrm{f} \Lambda \mathrm{CDM}$ is $\Delta \chi^{2} \approx 4$ while for SNLS the excess is $\Delta \chi^{2} \approx 9$ (see Fig. (1).

A negatively curved geometry elongates the CMB distance and allows a low $\Omega_{m}$ in agreement with the SN distances [16, 30]. Even so, the CMB constraint on $\Omega_{m} h^{2}$ favors a high Hubble constant of $h=0.8$ (SNGold) and $h \approx 0.9$ (SNLS) compared with observations from the HST Key Project (KP) $h=0.72 \pm 0.08$ [31]. f $\Lambda \mathrm{CDM}$ on the other hand, despite being highly over-constrained satisfies the observations with $h=0.73$.

With the addition of the $\mathrm{KP} H_{0}$ constraint, the best fit open DGP (oDGP) model has $h=0.76$ (SNGold) and 
$h=0.82$ (SNLS) but is a marginally poorer fit to the data than $\mathrm{f} \Lambda \mathrm{CDM}$. The excess over $\mathrm{f} \Lambda \mathrm{CDM}$ of the latter is $\Delta \chi^{2} \sim 2.5$ with one fewer degree of freedom due to the fit to spatial curvature. The other parameters of this best fit model are $\Omega_{m}=0.18, \Omega_{k}=0.036$.

If the Hubble constant measurements can be improved by a factor of 2 in the future, then the oDGP and $f \Lambda C D M$ will be clearly distinguished from each other. They are currently both acceptable fits to these data. We will hereafter take the best fit model to the WMAP, SNLS and KP data sets as the oDGP model for further tests.

\section{B. DGP vs. Dark Energy}

The main expansion history differences between the best fit oDGP model and $\mathrm{f} \Lambda \mathrm{CDM}$ can be exposed by finding the scalar field or QCDM model that matches the oDGP distance measures exactly. With the same curvature, $\Omega_{m} h^{2}$ and $H_{0}$, that QCDM model has a timedependent equation of state

$$
w(a)=-\frac{1}{3} \frac{\frac{d E^{2}}{d \ln a}+3 \Omega_{m} a^{-3}+2 \Omega_{k} a^{-2}+4 \Omega_{r} a^{-4}}{E^{2}-\Omega_{m} a^{-3}-\Omega_{k} a^{-2}-\Omega_{r} a^{-4}}-1 .(6)
$$

This equation of state starts at $w=-1 / 3$ during radiation domination, becomes $w=-0.5$ during matter domination and decays to $w=-0.85$ at the present time. The value of $w(a)$ is substantially higher than the $w=-1$ of $\mathrm{f} \Lambda \mathrm{CDM}$. The implied increase in the dark energy density causes both the larger Hubble constant and a decrement in $D(z)$ at high redshift. This decrement is compensated by negative curvature to match the $\mathrm{CMB}$ distance.

The expansion history of this QCDM model is strikingly different from $\mathrm{f} \Lambda \mathrm{CDM}$ despite the fact that they both satisfy CMB and SN constraints. Both the increased dark energy density and the negative spatial curvature contribute to changes in structure formation that would violate even current observations. One therefore expects that structure formation tests should provide a strong constraint on the oDGP model. It is important to emphasize that it is not necessary to distinguish between oDGP and QCDM based on difference in the growth of structure. The comparison is between oDGP and $\mathrm{f} \Lambda \mathrm{CDM}$ because the QCDM model is not viable. We therefore use the degenerate QCDM model as a guide to understand the qualitative predictions of oDGP. The main task of testing the expansion history consequences for oDGP is to find a structure formation probe that can be robustly calculated in the DGP model.

\section{STRUCTURE FORMATION}

\section{A. Baryon acoustic oscillations}

As discussed in the previous section, the largest expansion history discrepancy between oDGP and $\mathrm{f} \Lambda \mathrm{CDM}$ lies in the current expansion rate or Hubble constant. In the distance-degenerate QCDM model, the large Hubble constant would be in strong conflict with the measured baryon acoustic oscillations in the SDSS galaxy correlations. Baryon acoustic oscillations appear near the scale where density perturbations become non-linear and hence cannot be considered a purely geometric test. Detailed predictions in the oDGP model below this scale require the solution of the $N$-body problem with a nonlinear Poisson equation. It is nonetheless useful to quantify the extent of the problem in the QCDM model since it determines the level at which nonlinear oDGP corrections would have to alter linear theory predictions to satisfy the data [16].

The baryon oscillations are imprinted at a fixed physical scale by the CMB at recombination. Under ordinary gravity, they yield a nearly geometric test since nonlinearities are insufficient to change the physical scale of features substantially. The SDSS luminous red galaxy (LRG) survey measures the features between $0.16 \leq$ $z \leq 0.47$. At these redshifts, this distance is sensitive mainly to the Hubble constant 32]. More specifically, it can be converted to a relative distance measure between the effective LRG redshift and recombination of $D(z=0.35) / D\left(z_{\mathrm{lss}}\right)=0.0979 \pm 0.0036$. Recall that the CMB measures $D\left(z_{\text {lss }}\right) / h$. Hence in the high Hubble constant QCDM model this number is too low, specifically 0.0818 leading to a $4.5 \sigma$ discrepancy. This discrepancy can be slightly ameliorated by refitting the models to all data including the BAO data [16].

If oDGP shared the same non-linear effects with QCDM, the BAO measurement would exclude it as well. In the DGP model, there is a key distance scale, $r_{*}$, above which the linearized perturbations experience the weakbrane phase [2, 33, 34, 35, 36]. In the weak-brane regime $r_{c} \gg r \gg r_{*}$, gravity is modified as a scalar-tensor theory. Below $r_{*}$, gravity is described by the standard Einstein theory. For a point mass $M$, the transition scale

$$
r_{*}=\left(r_{\mathrm{c}}^{2} r_{\mathrm{g}}\right)^{1 / 3}, \quad\left(r_{\mathrm{g}}=2 G_{\mathrm{N}} M\right) .
$$

Since even the linear density field is constructed from the spatial average of collapsed dark matter halos under any hierarchical structure formation model, the critical scale is associated with the $r_{*}$ of the dark matter halos.

For definiteness let us take the other cosmological parameters as in the best fit QCDM model: $\Omega_{m} h^{2}=0.122$, $\Omega_{b} h^{2}=0.0224, \tau=0.11$ with a power law spectrum of adiabatic fluctuations with $n_{s}=0.958, \delta_{\zeta}=4.5 \times 10^{-5}$ at $k_{\text {norm }}=0.05 \mathrm{Mpc}^{-1}$. In this QCDM model, the LRG galaxy number densities correspond to halos of $M_{*} \approx 10^{13} h^{-1} M_{\odot}$ corresponding to $r_{*} \approx 3 \mathrm{Mpc}$. This scale is only marginally smaller than the baryon oscillation scale. Furthermore, without a determination of how dark matter halos form under DGP and whether the observed galaxies indeed reside in $10^{13} h^{-1} M_{\odot}$ halos it remains unclear whether weak-brane phase predictions can be applied to the BAO measurements. 


\section{B. Linear Growth Rate}

The same expansion history discrepancy between QCDM and f $\Lambda$ CDM which raises $h$ also causes both an earlier decay of the growth of structure and a larger total change in the growth to $z=0$. For example, in the QCDM model $\sigma_{8}=0.6$ and $\Omega_{m}=0.18$. These values are in conflict with the abundance of local clusters. Unfortunately, this QCDM prediction again relies on the non-linear aspects of structure formation and hence cannot yet be applied to oDGP. In order to test oDGP we must find a probe on scales $r_{c} \gg r \gg r_{*}$.

In this regime, the linear theory predictions of DGP are now well understood. In general, the equations of motion of linear perturbations in DGP are not closed on the brane. They require knowledge of the gradient of metric perturbations into the bulk as quantified by the so-called master variable 37]. This in turn depends on boundary conditions in the bulk. Koyama and Maartens [13] solved this system in the small scale limit $k / a H \gg 1$ where the expansion rate can be ignored compared to the master variable frequency $\omega \sim k$. In this quasi-static (QS) limit, the Newtonian metric perturbations become

$$
\begin{aligned}
& \frac{k^{2}}{a^{2}} \Phi_{-}=4 \pi G \rho_{m} \Delta_{m}, \\
& \frac{k^{2}}{a^{2}} \Phi_{+}=-\frac{4 \pi G}{3 \beta} \rho_{m} \Delta_{m}
\end{aligned}
$$

where $\Phi_{-}=(\Phi-\Psi) / 2$ and $\Phi_{+}=(\Phi+\Psi) / 2$ and

$$
\beta=1-2 r_{c} H\left(1+\frac{\dot{H}}{3 H^{2}}\right) \text {. }
$$

Here $\Delta_{m}$ is the comoving matter density perturbation and for $k / a H \gg 1$ satisfies

$$
\ddot{\Delta}_{m}+2 H \dot{\Delta}_{m}=-\frac{k^{2}}{a^{2}}\left(\Phi_{+}-\Phi_{-}\right) .
$$

These relations agree with the earlier conclusions of Lue [5] obtained in a different manner.

This QS solution implies a 5\% to $10 \%$ extra decay of $\Phi_{-}$in the oDGP compared with the QCDM model with the same expansion history. We show the evolution of $\Phi_{-}$for each model in Fig. 2. The main effect however is not a difference between oDGP and QCDM but between either and $\mathrm{f} \Lambda \mathrm{CDM}$. In either case, $\Phi_{-}$begins to decay at high- $z$ such that there is as much decay at $z>1$ as there is in $\mathrm{f} \Lambda \mathrm{CDM}$ at $z<1$.

In order to find an appropriate test of this prediction, we need to determine the validity of the quasistatic solution on large scales approaching $r_{c}$. In a companion paper [38], we solved the dynamical equations by making a starting assumption that outside the horizon the evolution is scale free and that perturbations in the bulk vanish at the causal horizon. We then iterate the solution through the dynamical equations until they reach convergence. The starting scaling assumption is mildly violated during the transition from matter domination to accelerated expansion but the iteration yields stable solutions. We call this the dynamical scaling (DS) solution.

Under these assumptions the quasistatic solution is stably approached by $k=10 a H$ and hence is valid to better than $2 \%$ at $k \gtrsim 0.01 \mathrm{Mpc}^{-1}$ for $z=0$. Combined with the condition from $r_{*}$, a robust test of the quasi-static predictions of oDGP would involve fluctuations between $0.01 \lesssim k\left(\mathrm{Mpc}^{-1}\right) \lesssim 0.1$.

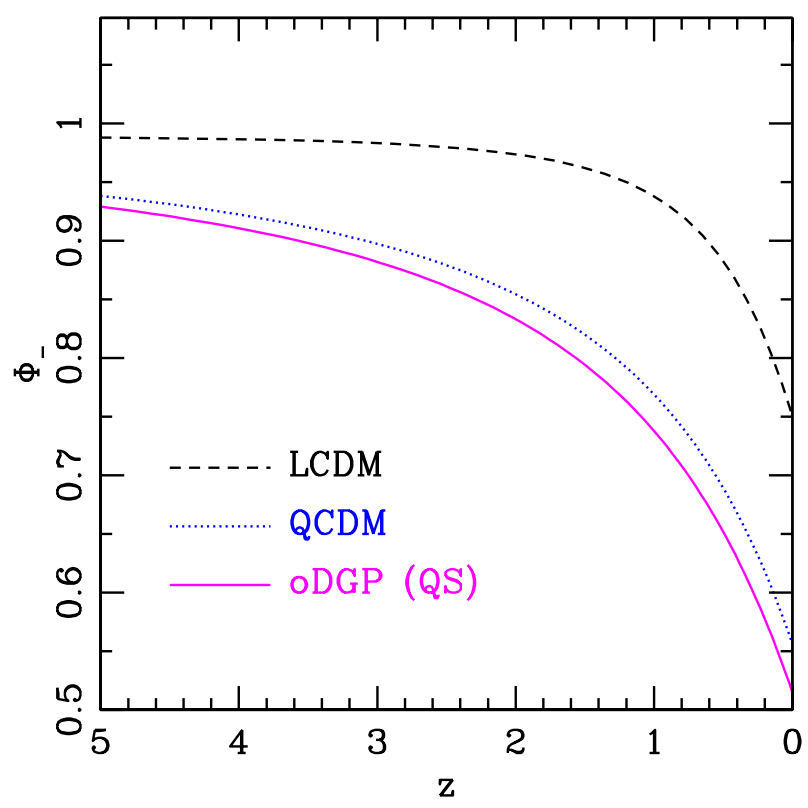

FIG. 2: The growth factor of $\Phi_{-}$of $\mathrm{f} \Lambda \mathrm{CDM}$ and oDGP under the quasi-static (QS) assumption normalized to $\Phi_{-}(z \gg 1)=1$. The model parameters are chosen as the best fit to the CMB (3yrWMAP), SN (SNLS), and $H_{0}(\mathrm{KP})$. QCDM is the scalar field dark energy model which has the same expansion history as oDGP. The growth rate is plotted on subhorizon scales where the dark energy is smooth and oDGP follows the QS assumption.

\section{ISW Effect}

The decay in the gravitational potential $\Phi_{-}$at $k \gtrsim$ $0.005 \mathrm{Mpc}^{-1}$ causes an enhancement of the large-angle anisotropy in the CMB through the ISW effect.

The angular power spectrum of the ISW effect is given by

$$
C_{l}^{\mathrm{II}}=4 \pi \int \frac{\mathrm{d} k}{k}\left[I_{l}^{\mathrm{I}}(k)\right]^{2} \frac{k^{3} P_{\Phi_{-} \Phi_{-}}(k, 0)}{2 \pi^{2}},
$$

where $P_{\Phi_{-} \Phi_{-}}(k, 0)$ is the power spectrum of $\Phi_{-}$at the present time and the kernel $I_{l}^{\mathrm{I}}$ is

$$
I_{l}^{\mathrm{I}}(k)=\int \mathrm{d} z \frac{\Phi_{-}(k, z)}{\Phi_{-}(k, 0)} W^{\mathrm{I}}(k, z) j_{l}(k D)\left(\frac{d D}{d r}\right)^{1 / 2} .
$$


The window function is given by

$$
W^{\mathrm{I}}(k, z)=\frac{2}{\Phi_{-}} \frac{\partial \Phi_{-}}{\partial z} .
$$

We have have here assumed that the spatial curvature is small enough that the spherical Bessel function $j_{l}$ accurately represents the radial harmonics.

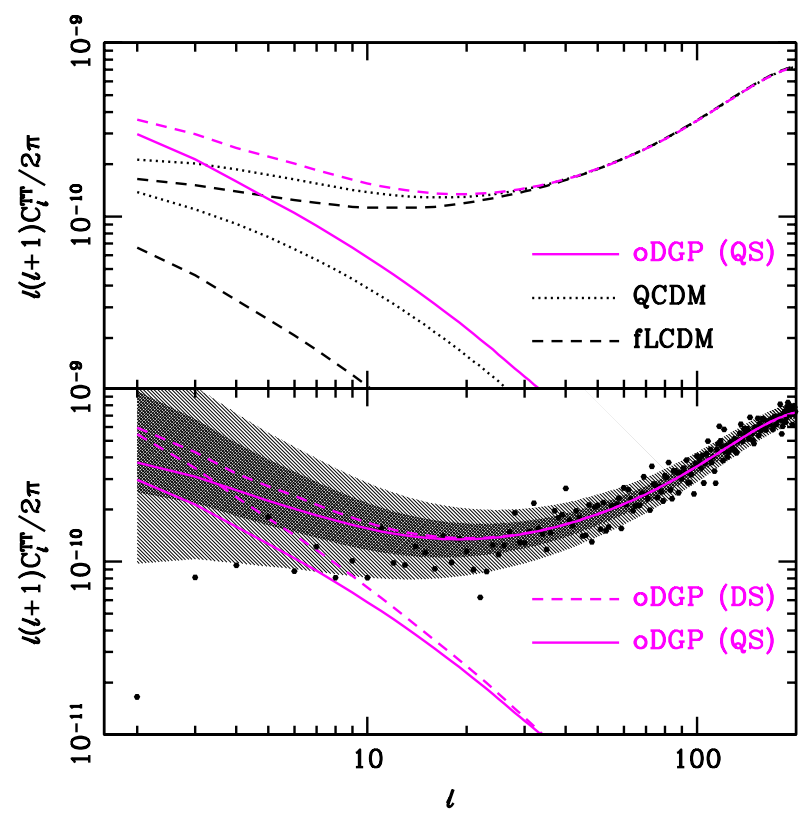

FIG. 3: The CMB temperature anisotropy power spectra of the various models. Top panel: oDGP under the quasi-static (QS) approximation versus QCDM and $\mathrm{f} \Lambda \mathrm{CDM}$ for the total power (upper curves) and the ISW effect (lower curves). Bottom panel: oDGP (QS) compared with the dynamical scaling (DS) solution. The DS solution predicts a further enhancement of the ISW effect such that $l<10$ strongly violates the WMAP measurements (points). The errors are mainly associated with cosmic variance at these multipoles and the $68 \%$ and $95 \%$ CL bands per $l$ have been attached to the DS model.

Fig. [3 shows the difference between predictions for $C_{l}^{\mathrm{II}}$ from oDGP under the quasistatic approximation compared with $\mathrm{f} \Lambda \mathrm{CDM}$ and QCDM. We also show the total temperature power spectrum $C_{l}^{\mathrm{TT}}$. The enhancement of the low-multipole power spectrum directly reflects the high-redshift decay of the gravitational potential in oDGP and QCDM. In QCDM the effect at the lowest multipoles is smaller since a scalar field has a Jeans scale at the horizon. Beyond this scale, dark energy perturbations slow the decay of the potential.

There are analogous corrections for oDGP on large scales but they have the opposite sign. Under the DS approximation the decay rate of the $\Phi_{-}$actually increases at the largest scales (see Fig. (4) leading to even larger fluctuations. In [38], we show that this approximation should at least give the right sign of the effect. The QS solution should thus be taken as a lower bound on the ISW effect. Hence the discrepancy between the oDGP
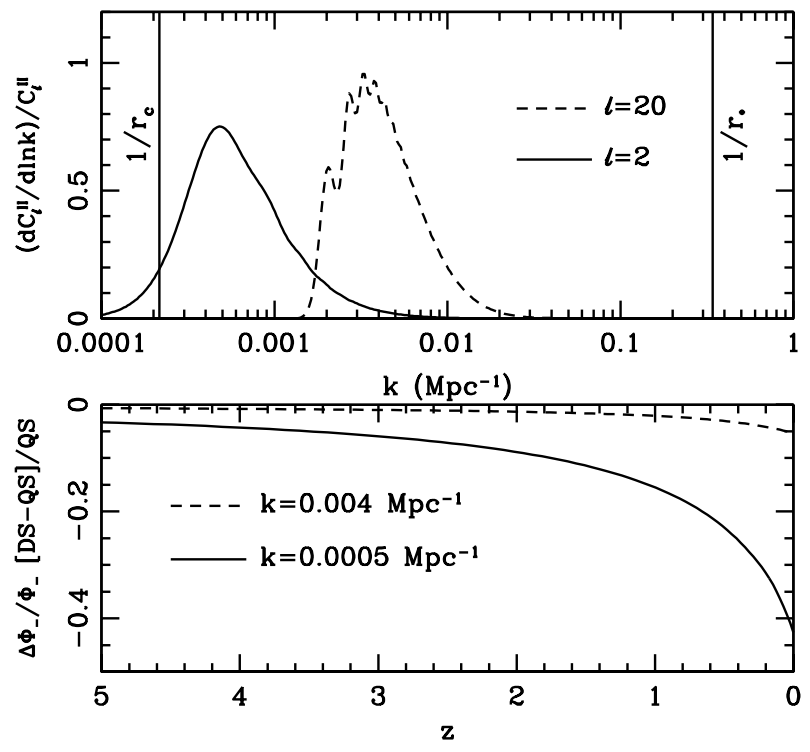

FIG. 4: Top panel: the spatial wavenumbers corresponding to the ISW effect under the QS approximation for multipoles $l=2,20$. For $l=20$ the scales associated with the effect are $1 / r_{c} \ll k \ll 1 / r_{*}$ and even the quadrupole receives much of its contribution from $1 / r_{c} \ll k$. Bottom panel: the fractional difference between the dynamical scaling (DS) and quasi-static (QS) solutions for DGP at the relevant scales for $l=2,20$. For $l=20$, the QS approximation is accurate to the several percent level.

QS predictions and the measurements at $l=2-5$ in the 3year WMAP data are a challenge to the model that will require either a cut off in the initial spectrum or other new phenomena near $r_{c}$ to overcome.

At $l \sim 20$ the ISW effect comes from physical scales that are within the limit of the quasistatic approximation as well as well below $r_{c}$. The ISW effect here is a small fraction of the total anisotropy and is best isolated through cross correlation with tracers of the gravitational potential, e.g. galaxies. Moreover the cross correlation can isolate the redshift history of the potential decay and expose a key prediction of the oDGP structure formation: an early decay of the potential.

\section{GALAXY-ISW CORRELATION TEST}

The oDGP model predicts a stronger and earlier decay of the gravitational potential than the $\mathrm{f} \Lambda \mathrm{CDM}$ model. By cross-correlating galaxies of different redshifts with the $\mathrm{CMB}$, one can in principle reconstruct the redshift history of the potential decay. Furthermore, the crosscorrelation arises from the well understood quasi-static (QS) regime of oDGP.

The cross-power spectrum of the CMB and a set of 


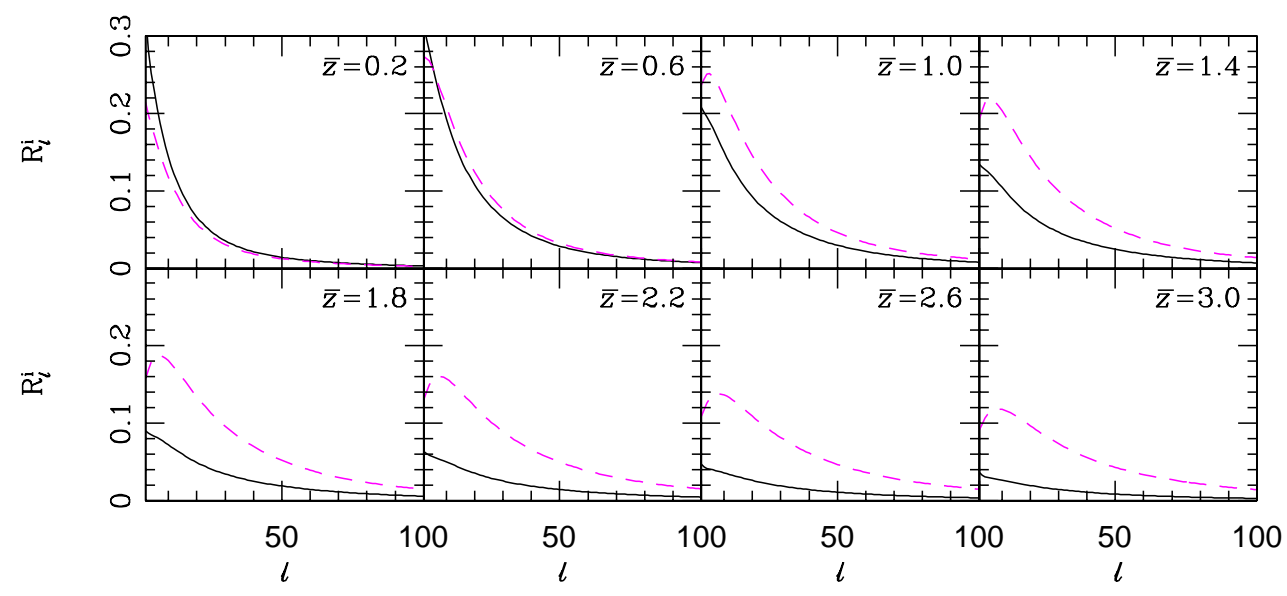

FIG. 5: The galaxy-ISW cross-correlation coefficient $R_{l}^{i}$ in each galaxy bin from $z=0$ to $z=3$. Solid curves denotes f $\Lambda$ CDM and dash curves denotes oDGP. Note the much larger correlation at high $z$ in oDGP.

galaxies $g_{i}$ is given by a generalization of Eq. (11)

$$
C_{l}^{g_{i} \mathrm{I}}=4 \pi \int \frac{\mathrm{d} k}{k} I_{l}^{g_{i}}(k) I_{l}^{\mathrm{I}}(k) \frac{k^{3} P_{\Phi_{-} \Phi_{-}}(k, 0)}{2 \pi^{2}},
$$

where the galaxy kernel $I^{g_{i}}$ is

$$
I_{l}^{g_{i}}(k)=\int \mathrm{d} z \frac{\Phi_{-}(k, z)}{\Phi_{-}(k, 0)} W^{g_{i}}(k, z) j_{l}(k D)\left(\frac{d D}{d r}\right)^{1 / 2}
$$

Under the QS approximation, the window function becomes

$$
W^{g_{i}}(k, z)=\frac{2}{3 \Omega_{m}} \frac{k^{2}}{H_{0}^{2}} \frac{n_{i}(z) b_{i}(z)}{1+z},
$$

where $n_{i}(z)$ is the redshift distribution of the galaxies normalized to $\int d z n_{i}=1$ and $b_{i}(z)$ is the galaxy bias.

For definiteness, we assume that the galaxy sets come from a net galaxy distributions of

$$
n_{g}(z) \propto z^{2} e^{-(z / 1.5)^{2}},
$$

where the normalization is given by the LSST expectation [42] of 35 galaxies per $\operatorname{arcmin}^{2}$. For the subsets of galaxies, we assume that this total distribution is separated by photometric redshifts which have a Gaussian error distribution with $\mathrm{rms} \sigma(z)=0.03(1+z)$ (see 39] for details). The redshift distributions are then given by

$n_{i}(z)=\frac{A_{i}}{2} n_{g}(z)\left[\operatorname{erfc}\left(\frac{z_{i-1}-z}{\sqrt{2} \sigma(z)}\right)-\operatorname{erfc}\left(\frac{z_{i}-z}{\sqrt{2} \sigma(z)}\right)\right]$,

where erfc is the complementary error function and $A_{i}$ is determined by the normalization constraint.

Our galaxy bias $b_{i}(z)$ is determined by a halo model and ranges from 1.4 at $z \sim 0$ to 3.8 at $z \sim 3$. However with our narrow binning, the bias is nearly constant across the bin and so its value can be empirically determined through comparing the auto and cross power spectra once the data are in hand. In particular, the cross-correlation coefficient $R_{l}^{i}$ in harmonic space

$$
R_{l}^{i}=\frac{C_{l}^{g_{i} \mathrm{I}}}{\sqrt{C_{l}^{\mathrm{TT}} C_{l}^{g_{i} g_{i}}}}
$$

is independent of the bias and is a robust measure of the galaxy-ISW correlation.

In Fig. 5 we show $R_{l}^{i}$ for oDGP and $\mathrm{f} \Lambda \mathrm{CDM}$. The main difference appears in the high-redshift bins and reflects the early decay of the potential in oDGP. The correlation of high-redshift galaxies with the CMB is therefore a sharp test of the oDGP model.

To determine whether these correlations are potentially observable, we estimate the sampling and noise errors in the cross-correlation measurement. First let us define the total galaxy power spectra $\tilde{C}_{l}^{g_{i} g_{i}}$ as

$$
\tilde{C}_{l}^{g_{i} g_{i}}=C_{l}^{g_{i} g_{i}}+N_{l}^{g_{i} g_{i}} .
$$

Here the shot noise $N_{l}^{g_{i} g_{i}}=1 / n_{A}^{i}$ where $n_{A}^{i}$ is the angular number density of galaxies in the bin. With our number densities the variance is nearly sample limited in all of the redshift bins.

Likewise the total power in the CMB measurements is given by

$$
\tilde{C}_{l}^{\mathrm{TT}}=C_{l}^{\mathrm{TT}}+N_{l}^{\mathrm{TT}}
$$

where the instrumental noise $N_{l}^{\mathrm{TT}}$ is $\Delta_{T}^{2} e^{l(l+1) \sigma^{2} / 8 \ln 2}$. With either WMAP or Planck satellite assumptions for the noise $\Delta_{T}$ and the beam $\sigma$ the temperature map is sample variance limited to good approximation.

The error on cross-correlation can be calculated under 
the Gaussian assumption as

$$
\Delta C_{l}^{g_{i} \mathrm{I}}=\sqrt{\frac{1}{(2 l+1) f_{\mathrm{sky}}}}\left[\left(C_{l}^{g_{i} \mathrm{I}}\right)^{2}+\tilde{C}_{l}^{g_{i} g_{i}} \tilde{C}_{l}^{\mathrm{TT}}\right]^{1 / 2},
$$

where $f_{\text {sky }}$ is the sky fraction covered by the galaxy survey.

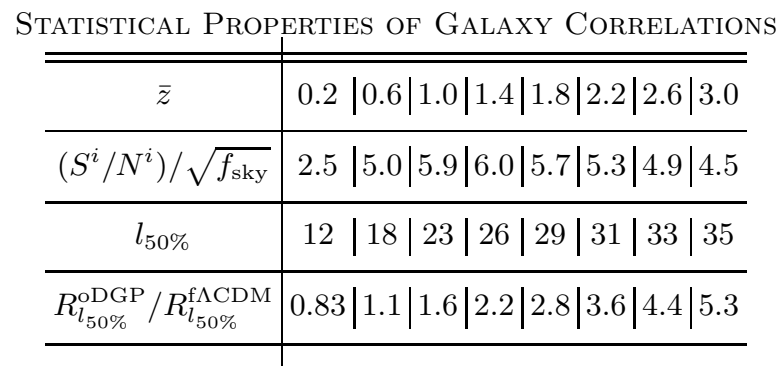

TABLE I: Rows: mean redshift, total signal-to-nose of correlation, multipole $l$ below which $50 \%$ of the $\left(S^{i} / N^{i}\right)^{2}$ arises, ratio of correlation between oDGP and $\mathrm{f} \Lambda \mathrm{CDM}$ at that multipole.

The signal to noise in each bin is given by

$$
\left(\frac{S^{i}}{N^{i}}\right)^{2}=\sum_{l}\left(\frac{C_{l}^{g_{i} \mathrm{I}}}{\Delta C_{l}^{g_{i} \mathrm{I}}}\right)^{2}
$$

and these values are given in Tab. I. In the sample variance limit approached by our fiducial assumptions

$$
\left(\frac{S^{i}}{N^{i}}\right)^{2} \approx \sum_{l}(2 l+1) f_{\mathrm{sky}} \frac{\left(R_{l}^{i}\right)^{2}}{1+\left(R_{l}^{i}\right)^{2}} .
$$

Even at high redshift $z \sim 2-3$ the signal to noise allows a $15 \%-20 \%$ measurement of the correlation. Such a measurement would be more than sufficient to distinguish the factor of a few difference between the oDGP and f $\Lambda$ CDM models.

Most of the detectable signal comes from the regime where the QS approximation is appropriate. At low multipoles $l<10$, the cosmic variance of both the galaxy and temperature fields dominate [40]. By $l=100$ the sample variance of the acoustic peaks dominates. From the signal to noise sum in Eq. (22), one can define $l_{50 \%}$ as the multipole at which the $\left(S^{i} / N^{i}\right)^{2}$ reaches half its total value. This scale is also given in Tab. \and lies between $l=20-40$ for most of the bins. At this scale the ratio of correlation coefficients in oDGP and $\mathrm{f} \Lambda \mathrm{CDM}$ approaches a factor of 6 at $z=3$. High redshift galaxies can thus test the quasi-static oDGP predictions with high significance.

\section{DISCUSSION}

The self-accelerating DGP braneworld model is challenged by expansion history constraints from the CMB, supernovae and the Hubble constant. Unlike a model with a cosmological constant as the dark energy, the DGP model requires spatial curvature to satisfy the $\mathrm{CMB}$ and supernovae constraint and even so mildly exceeds bounds on the Hubble constant.

In a dark energy model, the DGP expansion history would be ruled out by structure formation tests. These are serious but perhaps not insurmountable challenges for the model. Among the most powerful are the baryon oscillations and the large angle CMB anisotropy from the ISW effect. In the DGP model, these two phenomena lie close to the two critical scales: $r_{*}$ where gravity transitions from obeying the usual Einstein equations to a scalar-tensor or weak brane phase and $r_{c}$ when gravity exits the weak brane phase and becomes fully 5 dimensional. At the $r_{*}$ transition, the theoretical framework to calculate structure formation is currently incomplete. At the $r_{c}$ scale, changes in the initial power spectrum or new gravitational physics may ameliorate the problem.

The enhancement of the ISW effect from the decay of the gravitational potential extends to intermediate scales where the theoretical calculations are robust. The main difference between the DGP and the flat cosmological constant expansion history is the prediction that the gravitational begins its decay at high redshift. A sharp test of the DGP scenario would be to isolate this decay at high redshift by cross correlating the CMB with a high-redshift galaxy population. The cross correlation coefficient is also relatively robust to changes in the initial amplitude of fluctuations. We show that a galaxy survey that covers a substantial fraction of the sky at $z>1$ can detect this correlation at high significance. Such surveys are currently being planned for the measurement of highredshift baryon oscillations. Alternately, radio sources, $X$-ray sources and quasars provide other high-redshift populations for cross correlation.

A possible source of confusion for this test is the apparent number density fluctuation induced by magnification from gravitational lensing by the same structures associated with the ISW effect [41]. Magnification both lowers the number density for a fixed population behind a lens and also raises it for a fixed flux limit by bringing faint galaxies into the sample. This effect may be separated from a true spatial correlation by examining different populations of objects where the competition between these two effects differ and the cross-correlation between high and low redshift galaxy populations. A full examination of this issue is beyond the scope of this paper but it is likely that the galaxy-ISW cross correlation will become a sharp test of the DGP braneworld acceleration model in the future.

Acknowledgments: We thank Sean Carroll, Yu Gao, Nemanja Kaloper, Arthur Lue, and Xiaomin Wang for useful conversations. This work was supported by the U.S. Dept. of Energy contract DE-FG02-90ER-40560. IS and WH are additionally supported by the David and 
Lucile Packard Foundation. This work was carried out at the KICP under NSF PHY-0114422.
[1] G. R. Dvali, G. Gabadadze, and M. Porrati, Phys. Lett. B485, 208 (2000), hep-th/0005016.

[2] A. Lue, Phys. Rept. 423, 1 (2006), astro-ph/0510068.

[3] C. Deffayet, Phys. Lett. B502, 199 (2001), hepth/0010186.

[4] C. Deffayet, G. R. Dvali, and G. Gabadadze, Phys. Rev. D65, 044023 (2002), astro-ph/0105068.

[5] A. Lue, R. Scoccimarro, and G. D. Starkman, Phys. Rev. D69, 124015 (2004), astro-ph/0401515.

[6] Y.-S. Song, Phys. Rev. D71, 024026 (2005), astro$\mathrm{ph} / 0407489$.

[7] C. Schimd, J.-P. Uzan, and A. Riazuelo, Phys. Rev. D71, 083512 (2005), astro-ph/0412120.

[8] L. Knox, Y.-S. Song, and J. A. Tyson (2005), astro$\mathrm{ph} / 0503644$.

[9] M. Ishak, A. Upadhye, and D. N. Spergel (2005), astro$\mathrm{ph} / 0507184$.

[10] M. Amarzguioui, O. Elgaroy, D. F. Mota, and T. Multamaki (2005), astro-ph/0510519.

[11] E. V. Linder, Phys. Rev. D72, 043529 (2005), astro$\mathrm{ph} / 0507263$.

[12] I. Sawicki and S. M. Carroll (2005), astro-ph/0510364.

[13] K. Koyama and R. Maartens, JCAP 0601, 016 (2006), astro-ph/0511634.

[14] P. Zhang (2005), astro-ph/0512422.

[15] H. F. Stabenau and B. Jain (2006), astro-ph/0604038.

[16] R. Maartens and E. Majerotto (2006), astro-ph/0603353.

[17] D. N. Spergel et al. (2006), astro-ph/0603449.

[18] A. G. Riess et al. (Supernova Search Team), Astrophys. J. 607, 665 (2004), astro-ph/0402512.

[19] P. Astier et al., Astron. Astrophys. 447, 31 (2006), astro$\mathrm{ph} / 0510447$.

[20] D. J. Eisenstein et al. (2005), astro-ph/0501171.

[21] M. A. Luty, M. Porrati, and R. Rattazzi, JHEP 09, 029 (2003), hep-th/0303116.

[22] A. Nicolis and R. Rattazzi, JHEP 06, 059 (2004), hepth/0404159.

[23] D. Gorbunov, K. Koyama, and S. Sibiryakov, Phys. Rev. D73, 044016 (2006), hep-th/0512097.
[24] C. Charmousis, R. Gregory, N. Kaloper, and A. Padilla (2006), hep-th/0604086.

[25] C. Deffayet, G. Gabadadze, and A. Iglesias, JCAP 0608, 012 (2006), hep-th/0607099.

[26] A. G. Riess, A. V. Filippenko, P. Challis, A. Clocchiatti, A. Diercks, P. M. Garnavich, R. L. Gilliland, C. J. Hogan, S. Jha, R. P. Kirshner, et al., Astron. J. 116, 1009 (1998).

[27] S. Perlmutter et al. (Supernova Cosmology Project), Astrophys. J. 517, 565 (1999), astro-ph/9812133.

[28] S. Perlmutter, M. S. Turner, and M. White, Phys. Rev. Lett. 83, 670 (1999).

[29] D. N. Spergel et al. (WMAP), Astrophys. J. Suppl. 148, 175 (2003), astro-ph/0302209.

[30] M. Fairbairn and A. Goobar (2005), astro-ph/0511029.

[31] W. L. Freedman, B. F. Madore, B. K. Gibson, L. Ferrarese, D. D. Kelson, S. Sakai, J. R. Mould, R. C. Kennicutt, Jr., H. C. Ford, J. A. Graham, et al., Astrophys. J. 553, 47 (2001).

[32] D. J. Eisenstein, W. Hu, and M. Tegmark, Astrophys. J. Lett. 504, L57 (1998), astro-ph/9805239.

[33] A. Gruzinov, New Astron. 10, 311 (2005), astro$\mathrm{ph} / 0112246$.

[34] M. Porrati, Phys. Lett. B534, 209 (2002), hepth/0203014.

[35] A. Lue and G. Starkman, Phys. Rev. D67, 064002 (2003), astro-ph/0212083.

[36] G. Dvali, A. Gruzinov, and M. Zaldarriaga, Phys. Rev. D68, 024012 (2003), hep-ph/0212069.

[37] C. Deffayet, Phys. Rev. D66, 103504 (2002), hepth/0205084.

[38] I. Sawicki, Y.-S. Song, and W. Hu (2006), astro-ph/.

[39] W. Hu and R. Scranton, Phys. Rev. D70, 123002 (2004), astro-ph/0408456.

[40] N. Afshordi, Phys. Rev. D70, 083536 (2004), astroph/0401166.

[41] L. Hui, M. Loverde, and E. Gaztanaga (2006), in preparation.

[42] http://www.lsst.org 